\title{
Synthesis and Characterisation of Biologically Potent Novel Chalcone Moieties
}

\author{
MAHAMMADALI KHANUSIYA* and Z.M GADHAWALA \\ Department of Chemistry, The HNSB Ltd Science College, Himatnagar, Gujarat-India. \\ ${ }^{*}$ Corresponding author E-mail: khanusiya.mali@gmail.com
}

http://dx.doi.org/10.13005/ojc/320244

(Received: March 04, 2016; Accepted: April 20, 2016)

\begin{abstract}
As displaying a dominant biological interest of some amino chalcone derivatives which were synthesized by claisen-schmidt condensation reaction of amino acetophenone with aromatic aldehyde in presence of sodium hydroxide. These chalcones were screened for antifungal activity against candida albicans strain and also for antibacterial activity against staphylococcus epidermidis (G positive) and pseudomonas aeruginosa (G negative) strain by NCCLS method. The synthesized compounds were characterized by means of their FT-IR and ${ }^{1} \mathrm{HNMR}$ spectral study ${ }^{14}$.
\end{abstract}

Keywords: Amino chalcone, Antifungal, Antibacterial.

\section{INTRODUCTION}

Chalcones are pharmacologically valuable moieties possessing 1,3diphenyl prop-2-ene-1-one $(-\mathrm{CH}=\mathrm{CH}-\mathrm{CO}-)$ as a core structure in which two aromatic rings are linked by first and third carbon of a $\alpha, \beta$ - unsaturated carbonyl skeleton. A number of chalcone derivatives have demonstrated wide spectrum of pharmacological activities which has drawn the attention of medicinal chemist and pharmacologists. Due to the extended conjugation, the complete delocalisation of $\pi$ electrons on both the benzene rings makes whether from bioactivity aspect.
Isolation of Chalcone derivatives from nature requires a long and a far complicated procedure and comparable yield does not obtained. Chalcones and their derivatives are an interesting target class of compound which are extensively investigated due to their broad spectrum of various therapeutic activity such as antimicrobial ${ }^{1}$, anti-inflammatory ${ }^{2}$, antiulcerative ${ }^{3}$, antiviral ${ }^{4}$, antifungal ${ }^{5}$, antimalarial ${ }^{6,7}$, and anticancer ${ }^{8}$. Furthermore, chalcones are also known as the key intermediate in the synthesis of various biologically active heterocyclic compounds. In order to synthesize new therapeutic agents, this report illustrated the some novel amino chalcone derivatives and screening their activity against 
candila albicans fungi, gram positive and gram negative bacterial species.

\section{MATERIAL AND METHOD}

\section{General information}

The all starting materials and solvents were purchased from sigma-Aldrich and SD Fine and used without further purification. Melting points were determined by conventional method and then by electro capillary apparatus and are uncorrected. All the synthesized compounds were inspected by thin layer chromatography on silica gel (E-Merck) and the spots were identified by UV lamp.IR spectra and proton NMR spectra in DMSO at $500 \mathrm{MHz}$ were recorded at CSMCRI Bhavnagar.

\section{General procedure of synthesis of chalcone}

The synthesis of chalcone derivatives was conducted according to the procedure reported in the reference ${ }^{10-12}$. Acetophenone derivative (2.5milimole) and substituted benzaldehydes (2.5milimole) were dissolved in $30 \mathrm{ml}$ methanol. To the solution, 10 $\mathrm{ml} \mathrm{NaOH}(20 \%)$ solution was added drop wise and reaction mixture was stirred for 1-2 hour at room temperature by magnetic stirrer and kept for overnight. Subsequently, it was poured in ice water and neutralized. The solid precipitates was filtered off and recrystallized from methanol or ethyl acetate.

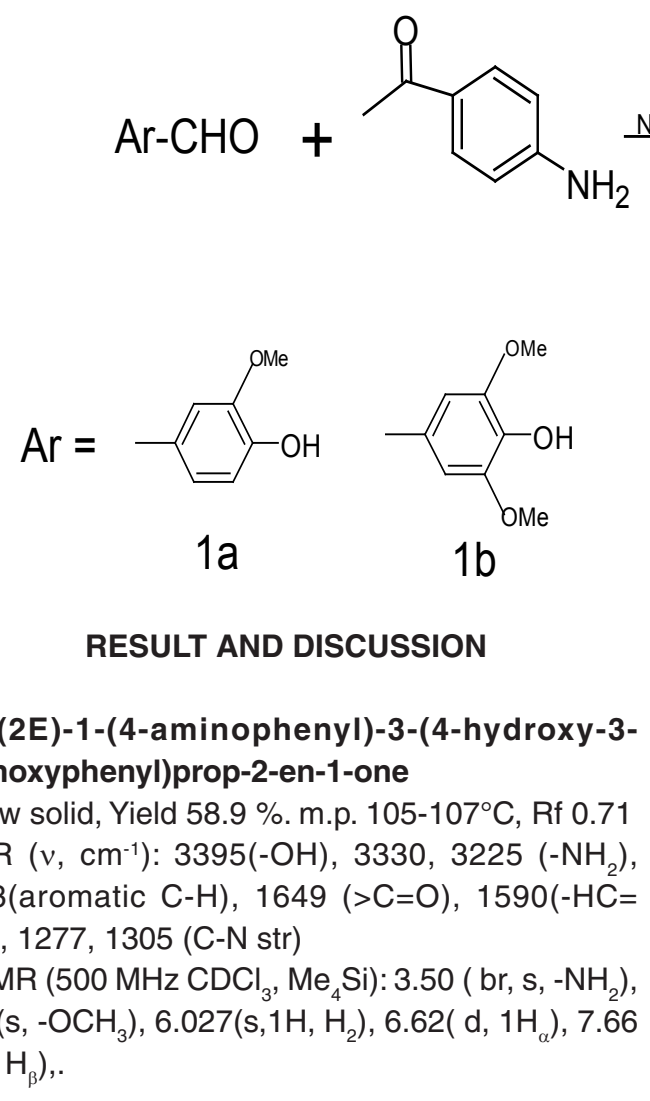

1b; (2E)-1-(4-aminophenyl)-3-(4-hydroxy-3,5dimethoxyphenyl)prop-2-en-1-one

Yellow solid, Yield $57.5 \%$. m.p. $98-100^{\circ} \mathrm{C}$, Rf 0.66 FT-IR $\left(v, \mathrm{~cm}^{-1}\right)$ : 3396(-OH), 3331, 3225 $\left(-\mathrm{NH}_{2}\right)$, 3064(aromatic C-H), $1648(>\mathrm{C}=\mathrm{O}), 1590(-\mathrm{HC}=$ $\mathrm{CH}-), 1276,1304$ (C-N str)<smiles>Cc1ccccc1O</smiles>

$1 \mathrm{c}$<smiles>Nc1ccc(C(=O)/C=C/[Al])cc1</smiles>

$1 a-1 e$<smiles>Cc1ccc(O)cc1O</smiles>

$1 d$<smiles>Cc1c(O)ccc2ccccc12</smiles>

$1 \mathrm{e}$
${ }^{1} \mathrm{HNMR}\left(500 \mathrm{MHz}\right.$ DMSO, $\left.\mathrm{Me}_{4} \mathrm{Si}\right): 3.49\left(\mathrm{br}, \mathrm{s},-\mathrm{NH}_{2}\right.$ ), 3.71(s, $\left.-\mathrm{OCH}_{3}\right), 3.82\left(\mathrm{~s},-\mathrm{OCH}_{3}\right), 6.028\left(\mathrm{~s}, 1 \mathrm{H}, \mathrm{H}_{1}, \mathrm{H}_{6}\right)$, 6.58( d, $\left.1 \mathrm{H}_{\alpha}\right), 7.68\left(\mathrm{~d}, 1 \mathrm{H}_{\beta}\right)$.

1c: (2E)-1-(4-aminophenyl)-3-(2-hydroxyphenyl) prop-2-en-1-one

Pale Yellow solid, Yield $62.5 \%$. m.p. $110-112^{\circ} \mathrm{C}$, Rf 0.68

FT-IR $\left(v, \mathrm{~cm}^{-1}\right): 3570(-\mathrm{OH}), 3338,3328\left(-\mathrm{NH}_{2}\right)$, 3050 (aromatic $\mathrm{C}-\mathrm{H}), 1676(>\mathrm{C}=\mathrm{O}), 1595(-\mathrm{HC}$ $=\mathrm{CH}-$ ), 1270,1364 (C-N str)

${ }^{1} \mathrm{HNMR}\left(500 \mathrm{MHz} \mathrm{CDCl}_{3}, \mathrm{Me}_{4} \mathrm{Si}\right): 3.47$ ( br, s, $\left.-\mathrm{NH}_{2}\right), 6.57\left(\mathrm{~d}, 1 \mathrm{H}_{\alpha}\right), 7.51\left(\mathrm{~d}, 1 \mathrm{H}_{\beta}\right), 7.013\left(\mathrm{~m}, \mathrm{H}_{2}\right)$, $7.72\left(\mathrm{~m}, \mathrm{H}_{3}, \mathrm{H}_{4}\right)$.

1d: (2E)-1-(4-aminophenyl)-3-(2,4-dihydroxy phenyl)prop-2-en-1-one

Yellow solid, Yield $87.0 \%$. m.p. $98-100^{\circ} \mathrm{C}$,Rf 0.72 
FT-IR ( $\left.v, \mathrm{~cm}^{-1}\right): 3680(-\mathrm{OH}), 3652(-\mathrm{OH}), 3370,3325$

$\left(-\mathrm{NH}_{2}\right)$, 3062(aromatic C-H), $1673(>\mathrm{C}=\mathrm{O}), 1596$ (-HC=CH-), 1270,1364 (C-N str)

${ }^{1} \mathrm{HNMR}\left(500 \mathrm{MHz} \mathrm{CDCl}_{3}, \mathrm{Me}_{4} \mathrm{Si}\right): 3.48(\mathrm{br}, \mathrm{s}$, $\left.-\mathrm{NH}_{2}\right), 6.32\left(\mathrm{~s}, 1 \mathrm{H}, \mathrm{H}_{2}\right), 6.55\left(\mathrm{~d}, 1 \mathrm{H}_{\alpha}\right), 7.48\left(\mathrm{~d}, 1 \mathrm{H}_{\beta}\right)$, $7.55\left(\mathrm{~d}, 1 \mathrm{H}, \mathrm{H}_{4}\right), 7.67\left(\mathrm{~d}, 1 \mathrm{H}, \mathrm{H}_{5}\right)$.

1e: (2E)-1-(4-aminophenyl)-3-(2-hydroxyna phthalen-1-yl) prop-2-en-1-one

Brick Red solid, Yield $73.6 \%$. m.p. $182-185^{\circ} \mathrm{C}$, Rf 0.53
FT-IR ( $\left.v, \mathrm{~cm}^{-1}\right): 3652(-\mathrm{OH}), 3322,3335\left(-\mathrm{NH}_{2}\right)$, 3044(aromatic $\mathrm{C}-\mathrm{H}), 1668(>\mathrm{C}=\mathrm{O}), 1587(-\mathrm{HC}=$ $\mathrm{CH}-), 1267,1349$ (C-N str)

${ }^{1} \mathrm{HNMR}\left(500 \mathrm{MHz} \mathrm{CDCl}_{3}, \mathrm{Me}_{4} \mathrm{Si}\right): 3.49$ ( br, s, $\left.-\mathrm{NH}_{2}\right), 6.58\left(\mathrm{~d}, 1 \mathrm{H}_{\alpha}\right), 7.68\left(\mathrm{~d}, 1 \mathrm{H}_{\beta}\right), 7.42(\mathrm{~m}, \mathrm{C}-\mathrm{H}$,$) ,$ 7.85(d,1H, $\left.\mathrm{H}_{3}\right), 8.05\left(\mathrm{~d}, 1 \mathrm{H}, \mathrm{H}_{2}\right)$.

\section{Antibacterial Study}

Gram Positive microorganism [Staphylococcus epidermidis]

The antibacterial activity performed by antimicrobial susceptibility tests, NCCLS 1993,

\section{Staphylococcus epidermidis}

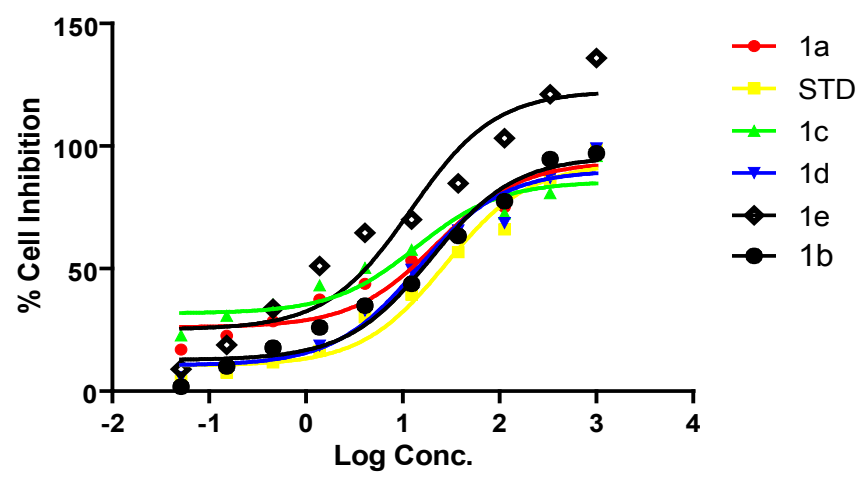

Fig. 1: Plot of log conc. $(\mu \mathrm{g}) \mathrm{v} / \mathrm{s} \%$ cell inhibition of compounds against Staphylococcus epidermidis

Table 1: Percentage cell inhibition by compounds agains Staphylococcus epidermidis strain

\begin{tabular}{lccccccc}
\hline $\begin{array}{l}\text { Conc. } \\
(\boldsymbol{\mu} \mathbf{g} / \mathrm{ml})\end{array}$ & Log Conc. & $\mathbf{1 a}$ & $\mathbf{1 b}$ & $\mathbf{1 c}$ & $\mathbf{1 d}$ & $\mathbf{1 e}$ & STD \\
\hline 0.01 & -2.29 & 1.023 & 12.23 & 1.08 & 11.02 & 7.16 & 10.12 \\
0.02 & -1.82 & 1.02 & 14.11 & 5.22 & 10.22 & 8.02 & 11.89 \\
0.05 & -1.34 & 4.05 & 17.02 & 7.13 & 14.74 & 9.11 & 13.98 \\
0.14 & -0.86 & 5.11 & 21.26 & 22.41 & 17.23 & 13.21 & 18.03 \\
0.41 & -0.39 & 11.23 & 26.17 & 30.27 & 28.21 & 15.42 & 28.49 \\
1.23 & 0.09 & 14.52 & 32.20 & 32.36 & 27.62 & 18.85 & 33.26 \\
3.70 & 0.57 & 25.34 & 39.19 & 42.87 & 40.33 & 25.63 & 42.13 \\
11.11 & 1.05 & 29.78 & 52.36 & 48.16 & 63.11 & 40.17 & 49.65 \\
33.33 & 1.52 & 37.41 & 68.23 & 49.20 & 74.42 & 67.32 & 70.32 \\
100.00 & 2.00 & 67.28 & 74.12 & 54.14 & 93.12 & 87.24 & 84.97 \\
$\mathrm{IC50} \mu \mathrm{g} / \mathrm{ml}$ & & 7.69 & 5.67 & 0.30 & 7.60 & 21.61 & 7.625 \\
$\mathrm{R}^{2}$ & & 0.9227 & 0.9760 & 0.9632 & 0.9773 & 0.9923 & 0.9507 \\
& & & & & & & \\
\hline
\end{tabular}


Approved standard: M2-A5. Chemically synthesized compounds were taken for antibacterial activity. P.aeruginosa (Gram negative), S.epidermidis (Gram positive) strains were used for the antibacterial activity. Three-fold serial dilutions of the compounds $(150 \mu l)$ of each sample were made in sterile broth (nutrient broth). The specified amount of test organisms $(50 \mu \mathrm{l})$ was added to each dilution to give a final volume of $200 \mu \mathrm{l}$. After incubation at $37^{\circ} \mathrm{C}$ for 18-24 $\mathrm{h}$ the plates were examined for growth of the organisms. Absorbance was read in a plate reader. The figures showed the plot of log concentration Vs \% cell inhibition of compound against tested organisms. Test compounds show the dose-effect co-relation with maximum linearity in almost all cases within 0.8321 to 0.9863 . The data analysis was accomplished using Graph Pad Prism version 5.00, Graph Pad Software Inc., San Diego California USA. IC $\mathrm{I}_{50}$ values were obtained from regression lines with coefficient factors between $\mathrm{R}^{2}=0.52$ and 0.99 . Absorbance at $595 \mathrm{~nm}$ between reading taken before and after incubation of the plates.

Results of gram positive organism inhibition study indicate followings compounds were found to be lid among others when it was compare $\mathrm{IC}_{50}$

\section{Pseudomonas aeruginosa}

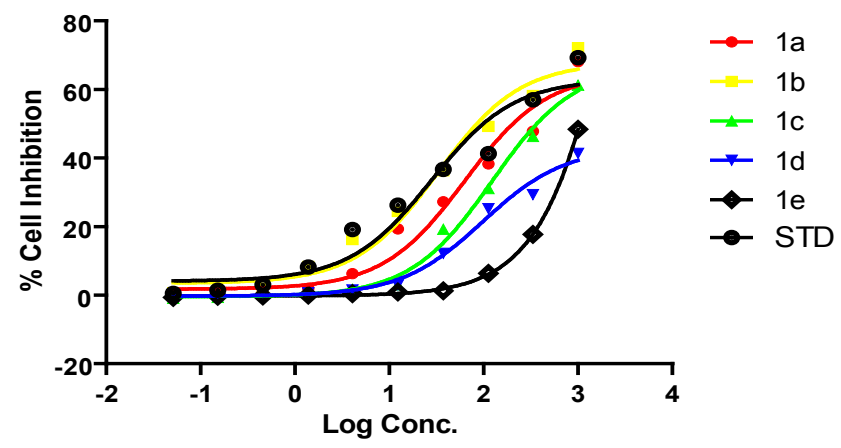

Fig. 2: Plot of log conc. $(\mu \mathrm{g}) \mathrm{v} / \mathrm{s} \%$ cell inhibition of compounds against Pseudomonas aeruginosa

Table 2: Percentage cell inhibition by compounds against Pseudomonas aeruginosa strain

\begin{tabular}{lccccccc}
\hline $\begin{array}{l}\text { Conc. } \\
(\boldsymbol{\mu} \mathbf{g} / \mathrm{ml})\end{array}$ & Log Conc. & $\mathbf{1 a}$ & $\mathbf{1 b}$ & $\mathbf{1 c}$ & $\mathbf{1 d}$ & $\mathbf{1 e}$ & STD \\
\hline 0.01 & -2.29 & 7.43 & 14.32 & 8.10 & 1.23 & 4.30 & 8.00 \\
0.02 & -1.82 & 10.22 & 15.26 & 12.42 & 2.52 & 7.12 & 12.94 \\
0.05 & -1.34 & 11.14 & 15.79 & 15.04 & 6.21 & 11.25 & 9.67 \\
0.14 & -0.86 & 17.16 & 20.64 & 22.16 & 5.11 & 13.02 & 13.41 \\
0.41 & -0.39 & 21.03 & 29.71 & 27.65 & 9.21 & 16.27 & 18.87 \\
1.23 & 0.09 & 22.17 & 32.27 & 35.44 & 16.23 & 19.15 & 23.42 \\
3.70 & 0.57 & 30.13 & 42.24 & 39.87 & 35.21 & 22.27 & 26.51 \\
11.11 & 1.05 & 42.16 & 48.24 & 45.31 & 39.47 & 35.65 & 29.04 \\
33.33 & 1.52 & 58.23 & 64.64 & 52.55 & 47.11 & 47.68 & 52.67 \\
100.00 & 2.00 & 64.12 & 83.12 & 81.65 & 54.20 & 77.24 & 76.19 \\
$\mathrm{IC} 50 \mu \mathrm{g} / \mathrm{ml}$ & & 7.908 & 3.795 & 10.30 & 2.795 & 34.94 & 9.871 \\
$\mathrm{R}^{2}$ & & 0.9737 & 0.9384 & 0.8675 & 0.9863 & 0.9643 & 0.9473 \\
& & & & & & & \\
\hline
\end{tabular}


value obtained by Streptomycin $(7.625 \mu \mathrm{M} / \mathrm{ml})$ with standard drug inhibitory value.

1c $\left(\mathrm{IC}_{50}\right.$ value; $\left.0.3086 \mu \mathrm{M} / \mathrm{ml}, \mathrm{R}^{2}=0.9632\right)>$ $1 \mathrm{~b}\left(\mathrm{IC}_{50}\right.$ value; $\left.5.303 \mu \mathrm{M} / \mathrm{ml}, \mathrm{R}^{2}=0.8821\right)>1 \mathrm{~d}>1 \mathrm{a}$

Results for gram negative microorganism, most effective compounds were arranged in following order when it was compare $\mathrm{IC}_{50}$ value obtained by Streptomycin $(9.871 \mu \mathrm{M} / \mathrm{ml})$ with standard drug inhibitory value:
$1 \mathrm{~d}\left(\mathrm{IC}_{50}\right.$ value; $\left.2.795 \mu \mathrm{M} / \mathrm{ml}, \mathrm{R}^{2}=0.9863\right)>1 \mathrm{~b}$ $\left(\mathrm{IC}_{50}\right.$ value; $\left.3.795 \mu \mathrm{M} / \mathrm{ml}, \mathrm{R}^{2}=0.9384\right)>1 \mathrm{a}\left(\mathrm{IC}_{50}\right.$ value; $\left.7.908 \mu \mathrm{M} / \mathrm{ml}, \mathrm{R}^{2}=0.9737\right)>1 \mathrm{c}\left(\mathrm{IC}_{50}\right.$ value; $9.871 \mu \mathrm{M} /$ $\left.\mathrm{ml}, \mathrm{R}^{2}=0.9636\right)$

Fluconazole and test compounds were tested against Candida albican sin dose dependent manner. Table showed that the high $\mathrm{IC}_{50}$ value of $1 \mathrm{e}$ was found against Candida albicans having $\left(\mathrm{IC}_{50}\right.$ : $74.37 \mu \mathrm{g} / \mathrm{ml})$. Comparative lower $\mathrm{IC}_{50}$ value was found with compounds $1 \mathrm{~b}$ against candida albicans $\left(\mathrm{IC}_{50}\right.$ :

\section{Candida albicans}

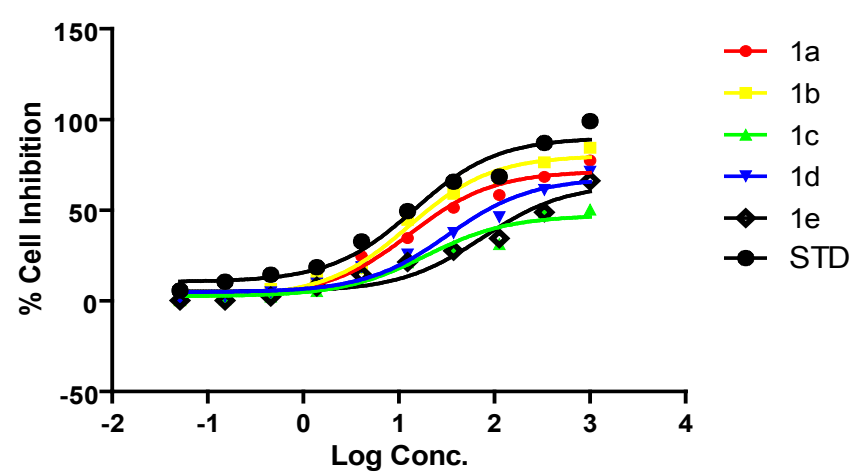

Fig. 3: Dose response curve of Test compounds against Candida albicans and Fluconazole

Table 3: Percentage growth inhibition of compounds against fungal strain and Fluconazole (Std.)

\begin{tabular}{lccccccc}
\hline $\begin{array}{l}\text { Conc. } \\
(\boldsymbol{\mu} \mathbf{g} / \mathbf{m l})\end{array}$ & Log Conc. & $\mathbf{1 a}$ & $\mathbf{1 b}$ & $\mathbf{1 c}$ & $\mathbf{1 d}$ & $\mathbf{1 e}$ & $\mathbf{S T D}$ \\
\hline 0.05 & -1.29 & -0.02 & 0.35 & 0.36 & -0.35 & 0.22 & 5.68 \\
0.15 & -0.82 & 0.032 & 2.36 & 0.89 & 2.35 & 0.156 & 10.64 \\
0.46 & -0.34 & 6.235 & 6.58 & 2.37 & 4.79 & 2.36 & 14.47 \\
1.37 & 0.14 & 11.35 & 13.26 & 5.46 & 9.63 & 7.86 & 18.63 \\
4.12 & 0.61 & 24.65 & 17.89 & 18.65 & 18.67 & 14.62 & 32.87 \\
12.35 & 1.09 & 34.68 & 44.65 & 21.34 & 25.63 & 21.35 & 49.58 \\
37.04 & 1.57 & 51.34 & 58.96 & 29.62 & 37.48 & 27.65 & 65.65 \\
111.11 & 2.05 & 58.36 & 67.25 & 31.28 & 46.22 & 34.52 & 68.61 \\
333.33 & 2.52 & 68.34 & 76.38 & 48.96 & 61.23 & 48.75 & 87.09 \\
1000.00 & 3.00 & $\mathbf{7 7 . 3 5}$ & 84.36 & 50.30 & 71.22 & 66.22 & 99.08 \\
$\mathbf{I C 5 0} \boldsymbol{\mu g} / \mathbf{m l}$ & & $\mathbf{1 3 . 1 2}$ & $\mathbf{1 2 . 6 9}$ & $\mathbf{1 9 . 0 2}$ & $\mathbf{3 2 . 2 9}$ & $\mathbf{7 4 . 3 7}$ & $\mathbf{1 4 . 3 9}$ \\
$\mathbf{R}^{2}$ & & $\mathbf{0 . 9 8 0 9}$ & $\mathbf{0 . 9 8 9 5}$ & $\mathbf{0 . 9 3 5 8}$ & $\mathbf{0 . 9 6 8 0}$ & $\mathbf{0 . 9 3 9 4}$ & $\mathbf{0 . 9 6 8 5}$ \\
& & & & & & & \\
\hline
\end{tabular}


$12.6 \mu \mathrm{g} / \mathrm{ml})$. The figure showed the plot of log concentration vs. \% cell inhibition of test compounds against Candida albicans. Test compounds show the dose-effect co-relation with maximum linearity $\left(R^{2}\right.$ value) with value being 0.9895 and comparatively lower linearity in case of Candida albicans at value being 0.9358 . Activity order of compounds can be summarized as : $1 b>1 a>1 c>1 d$.

\section{CONCLUSION}

From above results we can conclude that compounds $1 \mathrm{a}, 1 \mathrm{~b}$ and $1 \mathrm{c}$ are more effective in gram positive organism i.e. Staphylococcus epidermidis, While compounds like $1 \mathrm{a}, 1 \mathrm{~b}$ and $1 \mathrm{~d}$ are more effective against gram negative organism i.e. Pseudomonas aeruginosa.

Moreover, 1a and 1b both show good antifungal activity on Candida albicans strain, while $1 \mathrm{c}$ and $1 \mathrm{~d}$ having comparable activity and compound $1 \mathrm{e}$ is less effective.

\section{ACKNOWLEDGEMENT}

The author M M Khanusiya gratefully acknowledges CSIR, New Delhi for MANF. The authors are also expressing their sincere thanks to The HNSB Ltd Science College for performing research work and faculty of pharmacy, DDU Nadiad for antimicrobial tests.

\section{REFERENCES}

1. Prasad, Y.R; Kumar, P.R; Deepti, C.A; Ramna, M.V; E-Journal of chem, 2006, 3(3), 236241

2. Heish, H.K; Lee, T.H; Wang, J.J; Lin, C.N; Phasm. Res. 1998, 15(1), 39-46

3. Murakami, S; Muramatsu, M; Aihara, H; Otomo, S; Biochem. pharma, 1991,42(7), 1447-1451

4. Wu, J.H; Wang, X.H;Yi, YH; Lee, K.H; Bioorg. Med.Chem.Lett, 2003, 13(10), 1813-1815.

5. Lopaz, S.N; Castle, M.V; Jacchino, S.A; Dominguez, J.N; Lobo, G; Cortsey, J.C; Ribas, J.C; Devia, C; Rodrigues, A.M; Enriz, R.D; Bioorg. Med.Chem. 2001, 9(8), 19992004.

6. Leon, C; Gut, J; Rosenthal, P.J; J.Med. Chem.2005, 48, 3654.

7. Ram, V; Saxena, A; Srivastava, A; Bioorg.

Medicinal chemistry lett.2000, 10,2159

8. Liu, M; Wilairat, P; Crajt, S; Bioorg. Med. Chem. 2003, 11,2729.

9. Kumar, S.K; Hager, E; Pettit, C; Gurulingappa, H; Devidson,N.E; Khan, S.R; J. Med. Chem. 2003,46,2813-2815.

10. Suvito, $\mathrm{H}$; Jumina; Mustofa; Mutuzahroh , Ni; Nyoman, Ni; Der Pharma Chemica. 2015, 7(3), 89-94.

11. Ngameni, B; Kuete, V; Ambasa, P; Justine, K; Marlyse, M; Medicinal Chem 2013, 3; 233237.

12. Abdula A; European Journal of Chemistry 2013, 4(2), 207-210J.

13. Pngaew, R; Saekee, A; Mandi, P; Nantasenamat, C; Prachyasittikul, S; Ruchirawat ,S; Prachyasittikul, V; European Journal of Chemistry, 2014,85,65-76.

14. Khanusiya, M.M; Gadhawala Z.M; National Seminar on 'Frontier Areas In Chemical Sciences', 2016, 42. 